\title{
Volume 12 reviewers
}

The journal thanks the following people for their support in acting as reviewers of submitted papers.

\begin{tabular}{|c|c|c|c|}
\hline 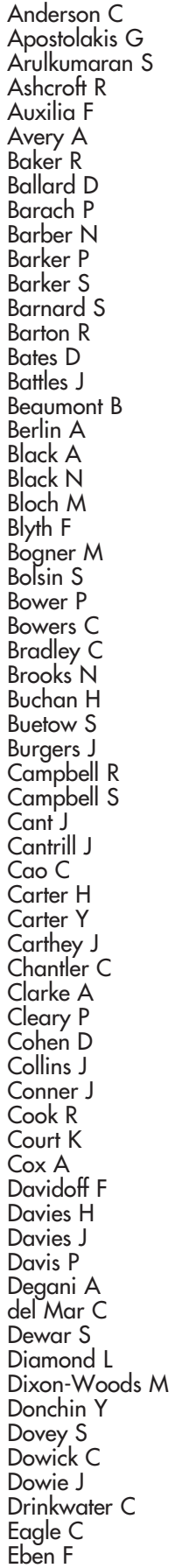 & $\begin{array}{l}\text { Edwards A } \\
\text { Ehrenwerth J } \\
\text { Elwyn G } \\
\text { Emslie S } \\
\text { Entwistle V } \\
\text { Ewings P } \\
\text { Federico F } \\
\text { Fenn P } \\
\text { Ferner R } \\
\text { Fielder A } \\
\text { Firth-Cozens J } \\
\text { Fisher J } \\
\text { Flanagan B } \\
\text { Flin R } \\
\text { Flottorp S } \\
\text { Ford G } \\
\text { Foy R } \\
\text { Frankel A } \\
\text { Franklin B } \\
\text { Franks A } \\
\text { Gaba D } \\
\text { Garside P } \\
\text { Gatta G } \\
\text { Gaylin D } \\
\text { Gelijins A } \\
\text { Gibberd R } \\
\text { Gilbody S } \\
\text { Girvin J } \\
\text { Gladman J } \\
\text { Glasper E } \\
\text { Godfrey M } \\
\text { Goode D } \\
\text { Gosbee J } \\
\text { Gough P } \\
\text { Grant A } \\
\text { Gray J } \\
\text { Grissinger M } \\
\text { Guerlain S } \\
\text { Haerter M } \\
\text { Haraden C } \\
\text { Harwood M } \\
\text { Henriksen K } \\
\text { Herxheimer A } \\
\text { Hollyoak V } \\
\text { Holm H } \\
\text { Holtedahl K } \\
\text { Hotvedt R } \\
\text { Howard S } \\
\text { Hughes C } \\
\text { Humphrey C } \\
\text { Humphris D } \\
\text { Hurst K } \\
\text { Hurwitz B } \\
\text { Hutchinson A } \\
\text { John N } \\
\text { Joshi M } \\
\text { Kaai T } \\
\text { Kai J } \\
\text { Kanki B } \\
\text { Kay L } \\
\text { Kazandiian V } \\
\text { Keeney S } \\
\text { Kelly P } \\
\text { Kendall K } \\
\text { Kand }\end{array}$ & $\begin{array}{l}\text { Kern T } \\
\text { Kitson A } \\
\text { Kobus D } \\
\text { Larsen P } \\
\text { Lavery G } \\
\text { Layde P } \\
\text { Leach D } \\
\text { Lelliott P } \\
\text { Lewis G } \\
\text { Lewis M } \\
\text { Liliford R } \\
\text { Lindberg C } \\
\text { Loeb J } \\
\text { Longo M } \\
\text { Lowdon I } \\
\text { Macbeth F } \\
\text { Madhok R } \\
\text { Maher E } \\
\text { Mäkelä M } \\
\text { Manna R } \\
\text { Mant J } \\
\text { Maresh M } \\
\text { Marshall M } \\
\text { Matthes N } \\
\text { McAdam R } \\
\text { McCarley N } \\
\text { McFetridge B } \\
\text { McKenna H } \\
\text { McKenna J } \\
\text { McKibben L } \\
\text { McManus I } \\
\text { McNaughton H } \\
\text { McPherson K } \\
\text { Miles J } \\
\text { Miles P } \\
\text { Miller D } \\
\text { Mohammed M } \\
\text { Mohr J } \\
\text { Moore K } \\
\text { Morgan M } \\
\text { Morlock L } \\
\text { Morrison J } \\
\text { Muir M } \\
\text { Murray W } \\
\text { Nargund V } \\
\text { Neale G } \\
\text { Nebeker J } \\
\text { Nielsen D } \\
\text { Noyes J } \\
\text { O'Dowd T } \\
\text { Oliver S } \\
\text { Patel V } \\
\text { Payne S } \\
\text { Philibert I } \\
\text { Pirmohamed M } \\
\text { Pringle M } \\
\text { Rafferty A } \\
\text { Rapport F } \\
\text { Rhydderch M } \\
\text { Rice N } \\
\text { Rodgers H } \\
\text { Rogers S } \\
\text { Roth E } \\
\text { Rowan K } \\
\text { Mowd }\end{array}$ & $\begin{array}{l}\text { Rudd A } \\
\text { Runciman W } \\
\text { Rutherford B } \\
\text { Ryan A } \\
\text { Ryan R } \\
\text { Saks K } \\
\text { Salas E } \\
\text { Savitz L } \\
\text { Schyve P } \\
\text { Seagull J } \\
\text { Shaw C } \\
\text { Sheaff R } \\
\text { Sheikh A } \\
\text { Shekelle P } \\
\text { Short T } \\
\text { Smith H } \\
\text { Smith T } \\
\text { Snooks H } \\
\text { Stacy R } \\
\text { stern C } \\
\text { Stevens D } \\
\text { Stewart F } \\
\text { Sullivan F } \\
\text { Sutcliffe K } \\
\text { Sutherland K } \\
\text { Svab I } \\
\text { Tattersall M } \\
\text { Taxis K } \\
\text { Thomadsen B } \\
\text { Thomas E } \\
\text { Thomas R } \\
\text { Topping A } \\
\text { Upton D } \\
\text { Veit C } \\
\text { Verheii T } \\
\text { Vidal-Alaball J } \\
\text { Vincent C } \\
\text { Vitense H } \\
\text { Walshe K } \\
\text { Wears R } \\
\text { Weingart S } \\
\text { Weinger M } \\
\text { Wensing M } \\
\text { West E } \\
\text { Westrum R } \\
\text { White M } \\
\text { White P } \\
\text { Whitty P } \\
\text { Wiffen P } \\
\text { Wiles R } \\
\text { Williams R } \\
\text { Williams S } \\
\text { Wilson K } \\
\text { Wilson P } \\
\text { Wilson R } \\
\text { Wilson T } \\
\text { Winkens R } \\
\text { Woods D } \\
\text { Wright J } \\
\text { Wynne H } \\
\text { Xiao Y }\end{array}$ \\
\hline
\end{tabular}

